\title{
An innovative media arts curriculum for lower elementary school (kindergarten through second grade), which integrates technology seamlessly into the classroom curriculum
}

\author{
S. Nuss \\ Brookside Elementary School/ Vlasic Early Childhood Center, \\ Cranbrook Educational Community, Michigan, USA
}

\section{Abstract}

A unique curriculum for lower elementary grades (kindergarten-through second grade) has been created using the original "Ideation Conceptualisation" model for technology integration created by the author, Dr. Shirley Nuss, Media Arts/Computer instructor, and former colleague, Kimberly Conely. Dr. Nuss has created the innovative curriculum for lower elementary students at Brookside Cranbrook and the Vlasic Early Childhood Center, the elementary divisions of Cranbrook Educational Community (www.cranbrook.edu), a private college preparatory day and boarding school in Bloomfield Hills, Michigan. Her curriculum addresses the need for appropriate technology introduction to lower elementary students, which fits seamlessly into the existing classroom curriculum and uses computer applications in place of packaged programs or integrated technology learning systems. This curriculum is also designed to meet the developmental needs and emerging skills of the lower elementary student and provides a means of authentic assessment shown to adapt easily to media projects. Dr. Nuss developed the curriculum after researching the use of technology with young children and was able to then adapt it to fit the "Ideation Conceptualisation Model" showing the efficacy of the model in designing a technology integrated curriculum for the K-2 elementary student as well as grades 3-5 which was presented in the author's earlier work, "A Model for Collaboration: Integrating Curriculum Technology Into the Elementary Media Arts and Studies" [6]. The curriculum encourages the child to engage is such media making activities as creating and publishing a classroom book, creating an interactive slide show with Kid Pix, (a paint program) and PowerPoint, the use of Claymation and Stop Motion Pro to make a simple animation, and using Microsoft's software, PhotoStory3, to construct a visual alphabet book using digital images taken by the children. Student work will be used throughout the paper to explain how the model is used to design projects that will fit into existing curriculum and how it might be used to design technology related curricula for lower elementary students globally.

Keywords: interdisciplinary, digital enhancement, emotional connectivity, metacognitive, ideation-conceptualization, developmental and emerging skills, authentic assessment, cognitive development, coded media language. 


\section{Introduction}

How to develop an integrated technology and media arts curriculum for lower elementary grades using the "Ideation Conceptualisation Model" [6] developed for designing technology integrated curriculum for upper elementary students is the challenge and the goal for this paper. The model, which will be discussed later, guides the instructor through a flexible meta-cognitive process for designing media projects, which are age-appropriate, authentic tasks fitting into the existing senior kindergarten through second grade curriculum. Consequently, technology will become important as a tool to re-enforce what the students are learning in the classroom and not a subject separated from the classroom curriculum to be viewed as entertainment. The media projects must meet the criteria for assessment for the lower elementary learner with emerging cognitive skills, developing fine motor skills, and the necessary skills in social interaction as well as practice in listening and following directions. A review of the literature using technology with young children early in their educational experience was conducted to see how computer use, as well as other digital media, is being used and integrated by others in the field of technology education.

\section{Studies on technology use in lower elementary grades}

In today's society, children are exposed to media from a very young age. According to Woodard and Gridina's Media in the home 2000: The fifth annual survey of parents and children, children between the ages of two and five are using the computer for an average of 27 minutes per day [12]. This might indicate that children come to their formal educational experience with some basic computer skills. However, as Bray and Lovely state in their paper, "Technology: It's Primary", Children learn outside school that technologies, especially computers are toys, not tools. Teachers need to start as early as possible preparing students to think of technology as a tool and a medium for showing what we know, think, or feel. Modeling the appropriate uses of technology in the classroom can be a key element to young learners by showing that the human is in charge of the machine and not the reverse [1]. Educators must begin to not only acknowledge the consequences of the 'digital revolution' and the ways it will affect a child's cognitive and cultural development in and outside of the classroom, but devise strategies that will have them learning to use it as a challenging and powerful productivity tool and not a game to be won or entertainment with no purpose. In A Celebration of Neurons: An Educator's Guide to the Human Brain, the author states "Technology is generally a delightful experience when we focus on activities that student brains enjoy doing and doing well, such as exploring concepts, creating metaphors and images, estimating and predicting, cooperating on group tasks, and discussing issues. Yet technology loses its lustre when we force students to do things their brains don't enjoy and do poorly such as memorization, repetition and reading and writing compressed content which can be the design for drill and practice 
software. Therefore computer use in the developing years should look at the motivation behind what the student brain enjoys doing [10]. Add to this the short attention span of the young child with overly repetitive tasks and a set of alternate strategies seems to emerge. Wartella and Jennings in their paper "Children and Computers: New Technology-Old Concerns", suggest a need for further research needed to help inform and sustain the creation of more quality content for children. They also state that the research being conducted today indicates that computer use can contribute to a child's self-perception and affect a child's socialization in a variety of ways in school and at home through group work or shared projects [11]. They also found observational research showing that young children usually turn to each other rather than to an adult when for computer advice even though an adult is available [2].

The International Standards for Technology Education, ISTE in collaboration with the United States Department of Education developed performance indicators with regards to technology for children in grades Pre-Kindergarten through second grade as they appear in their publication, National Educational Technology Standards for Students - Connecting Curriculum and Technology. They are as follows:

\section{Prior to the completion of Grade 2 students will:}

1. Use input devices (e.g., mouse, keyboard, remote control) and output devices (e.g., monitor, printer) to successfully operate computers, VCRs, audiotapes, and other technologies.

2. Use a variety of media and technology resources for directed and independent learning activities.

3. Communicate about technology using developmentally appropriate and accurate terminology.

4. Use developmentally appropriate multimedia resources, (e.g., interactive books, educational software, elementary multimedia encyclopedias) to support learning.

5. Work cooperatively and collaboratively with peers, family members, and others when using technology in the classroom.

6. Demonstrate positive social and ethical behaviours when using technology.

7. Practice responsible use of technology systems and software.

8. Create developmentally appropriate multimedia products with support from teachers, family members, or student partners.

9. Use technology resources (e.g., puzzles, logical thinking programs, writing tools, digital cameras, drawing tools) for problems solving, communication, and illustration of thoughts, ideas and stories.

10. Gather information and communicate with others using telecommunications, with support from teachers, family members, and student partners.

With a review of the literature complete the next step was to look at the Model and see how it adapted to the lower grades. 


\section{The Model - three instructional components}

\subsection{Ideation-conceptualisation process}

Each grade level project idea is conceptualised using a visual tool (figure 1) that is consistent with a students' grade level curriculum, and developmental skills (emotional maturity, small and gross motor skills, visual recognition skills, and tested sequential learning capabilities). The ideation-conceptualization step is grounded in current research pertaining to brain development and learning. Research shows that long-term memory, which often involves factual/label and location components, should be supplemented by an emotional component that binds and contributes to the overall learning experience. It is important to note that a six-year-old can readily remember the words to a commercial jingle and/or a sitcom theme song, but often has difficulty remembering things he/she is asked to memorize or recite. The emotional connections caused by continual exposure to commercials, video and computer games seem to trigger a memory response that is not consistent with the rote memorization practices indigenous to the educational setting. Robert Sylwester encourages educators to "help students begin to find relationships between the somewhat random, often trivial fact filled experiences of everyday life and the few enduring principles that define life-and then to help them create and constantly test the memory networks that solidify those relationships" [10]. When developing a project that uses pre-visualization techniques and technology, it is paramount to include an emotional connection experience to encourage and promote the development of the imprinted brain pathways.

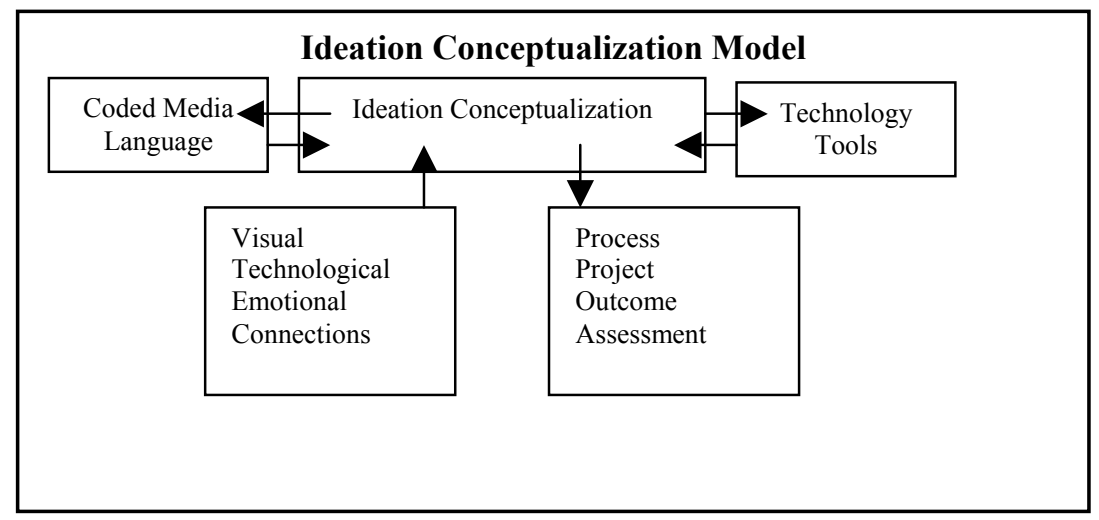

Figure 1: $\quad$ Ideation Conceptualization Model.

\subsection{Technology tools and coded medial language}

The second phase of our program incorporates comprehensive technological instruction with consideration of age appropriate fine and gross motor skills as well as maturation level ranges for the purposes of successfully executing grade 
specific projects. This includes all applicable media related terminology from, digital photography, printed media, specific computer programs related to the media projects and computer applications. Students are given multiple opportunities for hands-on experimentation with image capturing mechanisms and exposure to a wide variety of computer software applications. It is with this encouragement and additional hands-on instruction, that the younger students quickly address their fears and readily embrace the technology as a new form of personal expression and creativity.

\subsection{Processes, project and outcome}

The third step is total integration of phases one and two (listed above) supplemented by further exploration and development of creative thought processes resulting in a comprehensive, multi-disciplinary project. To effectively meet the stated goals, students are provided with the opportunity to create projects that they can claim ownership of, and share with their classmates and a wider audience. To help students learn to self-evaluate and edit, progress and project critiques are scheduled on a regular basis using the SmartBoard. There is no doubt that students seem genuinely captivated and motivated when they have the opportunity to create an original project using technology.

\subsection{Assessment of the media projects}

Media projects developed using the model require assessment using alternative assessment tools. These authentic projects are given an established set of criteria for assessment before the project begins and these are discussed with the children. What is expected and what constitutes a student's best effort whether individually or within a group is discussed with a very simple rubric that students can use for self-assessment. As stated above, the students use the Smart Board to present their projects. The presentation lends itself to positive critiquing with suggestions on what can be done to improve it. The third means of assessment is the final media product, which is sent home and shared with a larger audience of family and friends.

\section{The projects}

\subsection{Senior Kindergarten, First Grade and Second Grade Media Projects}

In addition to building upon age-appropriate cognitive skill levels, my goals for student learning and enrichment cover three basic developmental and learning areas. First, I want to provide a learning environment that enables the student to continuously hone the necessary skills required to use available technology as a means of expression and communication. The second objective is to familiarize the students with coded media language and help them learn to use their knowledge and skills as a basis for making informed decisions regarding their specific media choices. I provide ample opportunities for students to make and develop emotional connections by promoting the integration of topics, tools, and 
processes within their individualized project outcomes and to share these projects with a larger audience. A primary goal is to train the student to simultaneously see, conceive, create, emotionally connect with, and interpret his/her surroundings and experiences will use the technology to communicate their feelings and ideas about these connections which is consistent with what the research is saying about computer use with the young learner.

\subsubsection{Senior Kindergarten Project}

A popular classroom activity with the kindergartners in their classroom suggested a design for their project. It is the game "I Spy". This involves the teacher or a child picking an object which is visible to all in the classroom and the students asking ten "yes or no" questions and try to determine the object with questions that limit narrow the possibilities. The popular "I Spy" series of books for younger children is available in their library. A computer program, "I Spy Junior" (Scholastic) gives the students a screen image with a theme and asks the player to find certain objects identified using both text and auditory cues. After working on the program in the computer lab, the students are told that in the next few weeks they will be working in groups of two and creating their own "I Spy" page. They will use a scene with a theme, hide objects so they will be visible and shoot it with a digital camera. The project is discussed and a sample, created by the teacher, is shown to the students. While 3 groups of students are shooting different scenes, the remainder of the students continue working on the computer program while waiting their turn. Students receive camera instruction in small groups before capturing their images. Advance preparation for the activity includes setting up three specially lighted tables with free standing backgrounds based on a theme (e.g., Mother Goose, winter scenes, teddy bears, blocks, and favourite classroom books), a digital camera mounted at eye level on a tripod, and a box filled with smaller objects to be hidden but visible in the scene. The language of the media used is introduced as well as the simple parts of the camera. Students in small groups create and shoot their images, the images and inserted into PowerPoint by the teacher. They identify the hidden object to find using both text and recording it as a voice-over wav. file, which is inserted into the PowerPoint. Students print a copy of their slide to take home (figure 2). The media arts instructor, with the help of older students ( $5^{\text {th }}$ graders), creates a classroom "I Spy" using PowerPoint. The final project is burned on a $\mathrm{CD}$ for the students to take home.

A second semester project designed to fit the model is a Brown Bear...Brown Bear... What Do You See? classroom book, created by children, based on the popular children's' book by Bill Martin. The book is illustrated by each child using Kid Pix. First they draw a self-portrait of themselves and then they draw the object that the child sees "looking at me". The illustrations are exported from Kid Pix, in jpeg. Format, and inserted into a Word template designed for publishing a printable book. The book is printed (figure 3) and given to each child. It is read aloud in class with each child reading his/her page. The class uses the SmartBoard to critique it and the children take their books home to share with their family. 


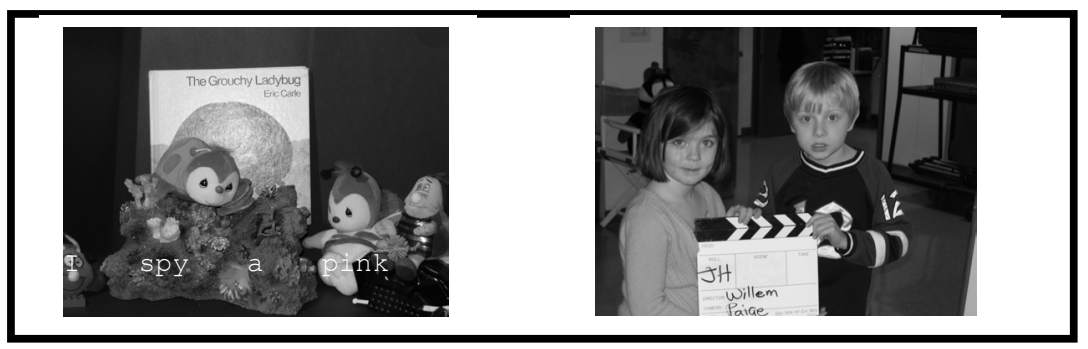

Figure 2: An "I Spy" page (using a favorite classroom book as a theme) and the senior kindergarten photographers who created the scene and took the digital image.

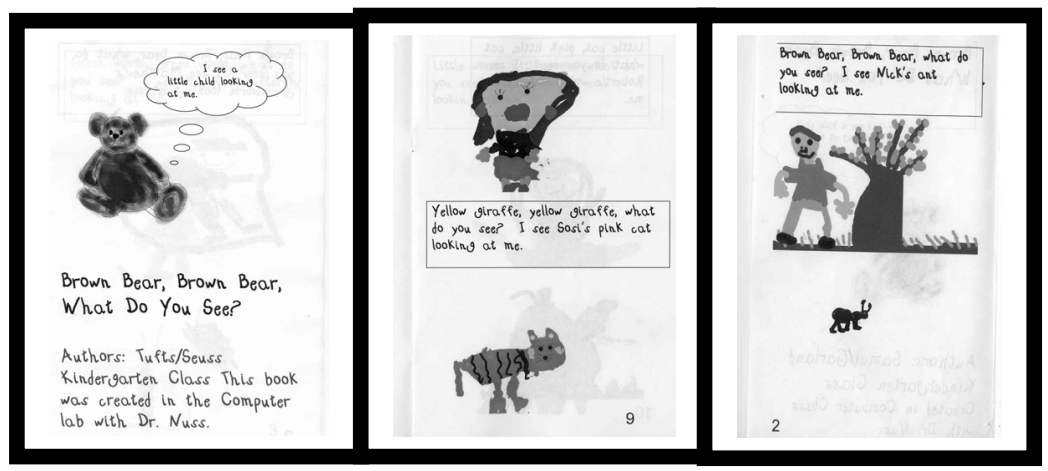

Figure 3: Brown Bear...Brown Bear book illustrated by the Senior Kindergarten.

How do these curricular activities follow the model? First the media language is identified, the Ideation-Conceptualisation phase allows for planning a visual project created with technology that they can form an emotional connection to because they have created something original, and it can be assessed with the alternative assessments designed for media projects.

\subsubsection{Grade 1}

The project, for first semester, focuses on the child's sense of emerging self. Students are taught camera shots and take a close up, medium, and wide shot of each other using the digital cameras. They draw a self-portrait of themselves in each of these shots using the paint program Kid Pix. Finally they draw a finished self-portrait, used as the basis for a book they will create, "About Me". The selfportraits are printed on note cards and arranged for class picture (figure 4). The book focuses on the child's favourite things, people, and activities. It features images drawn in the paint program and digital images taken by the child. 


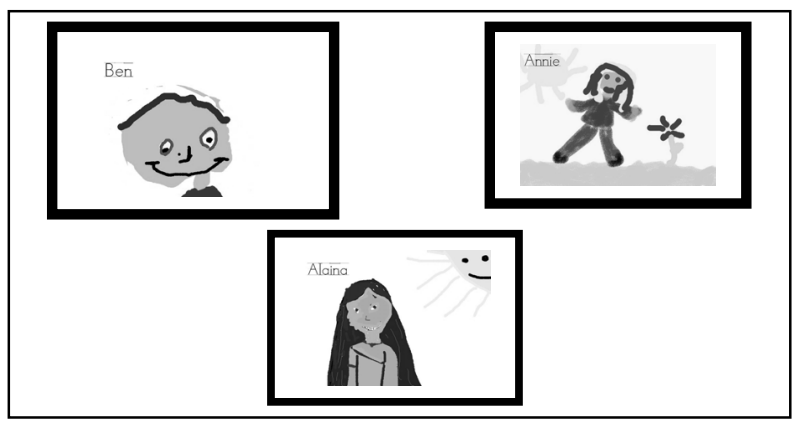

Figure 4: Close-up, medium, and wide shot for first grade self- portraits.

Second semester the students build on their camera skills with a claymation project. This introduces them to computer animation using student captured digital images. Students see that animation is created when the eye is tricked by a series of slightly different still shots referred to as "persistence of vision". After the introduction of the project, samples animations created by students in prior years are shown. Each student creates a simple character made of clay. In groups of two or three the students share the responsibilities of shooting or moving the clay figure for each shot. Either Claymation or Stop Motion Pro animation software is used to import the images and transform them into a simple animation saved as an avi. file. Students can add their own music or dialogue to the animation as options for the animating team to explore (figure 5).

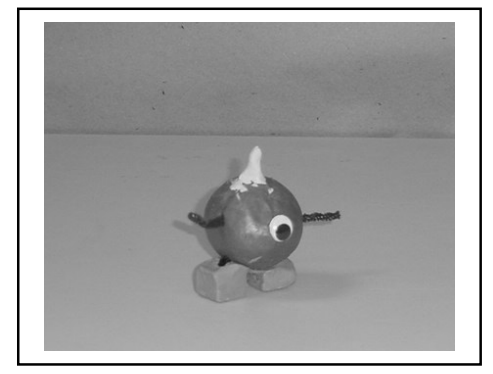

Figure 5: Student created clay character for first grade clay animation project.

\subsubsection{Grade 2}

The first semester begins with digital camera basics building upon the camera language and terms they learned in first grade. The students are given the chance to explore their world outside with a digital camera. They learn the coded media language relating to photo editing and image enhancement using Arc Soft Photo Studio, photo editing software. Their first project is to capture the Brookside Children's garden and then they have a variety of projects making art out of the 
photo images. These images are printed and taken home and are turned into notecards for our yearly fundraiser (figure 5).

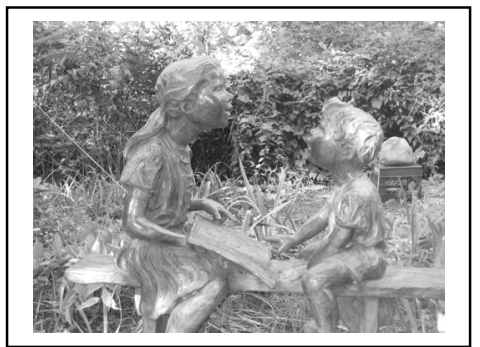

Figure 6: Student image of the Children's Garden at Brookside.

Second semester the students take digital images, working in groups of three, for a Digital Alphabet Book using Microsoft's PhotoStory3. This computer program takes the art of presentation software to a new level allowing for transitions, photo enhancements, and motion across the still photos. These projects can be saved as .wmv files and burned on a CD to be shared with other classes or taken home (figure 6).

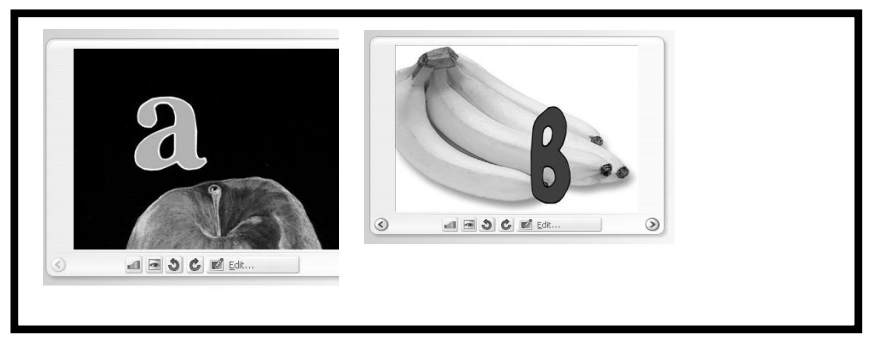

Figure 7: Student digital alphabet book created by second grade using Microsoft Photo Story 3. The alphabet book is saved as a .wmv file.

\section{Conclusion}

In conclusion, lower elementary school educators should strive to become more cognizant of ways computers and technology can be used with our youngest school children. They come to us knowing more about computers and media that we might suspect but their image of the computer is that of a pleasurable toy which serves no purpose other than to entertain. We need to show them that technology, computers and other media is a tool that enables them to express themselves creatively and effectively. This will give them the power to be knowledge producers as well as knowledge consumers and the skills to keep pace with the rapidly changing technology and media. Their world is greatly influenced by technology and is a world that heir parents and teachers could only dream of in their early education. Therefore, as teachers, we must do all we can 
to help them understand and use this valuable tool wisely for it is a tool invented and controlled by man.

\section{References}

[1] Bray, B. and Gail Lovely. Technology: It's Primary. Computer Using Educators. Technology and Learning. 2002 (http://techlearning.com)

[2] Clements, D.H. Computers and young children: A review of research. Young Children (1987) 43:34-44.

[3] Hyerle, David. Visual Tools for Constructing Knowledge. Alexandria, VA: Assoc. for Supervision and Curriculum Development, 1996.

[4] International Society for Technology in Education. National Educational Technology Standards for Students: Connecting Curriculum and Technology. U.S Department of Education and ISTE. 2000.

[5] Norton, Priscilla, and Karin M. Wiburg. Teaching With Technology: Designing Opportunities to Learn (2nd ed). Belmont, CA: Thomson Wadsworth, 2003.

[6] Nuss, S. and Kimberly Conely. A model for collaboration: integrating technology into the elementary media arts and studies curriculum. The Internet Society: Advances in Learning, Commerce, and Security Southhampton, Boston, WIT Press 2004.

[7] Orleans, M. and Laney, M.C. Children's computer use in the home; Isolation or socialization. Social Science Computer Review (Spring 2000) 18:56-72.

[8] Restak, Richard, M.D. The New Brain Modern Age is Rewiring Your Mind. Rodale Press, 2003.

[9] Simkins, Michael, Karen Cole, Fern Tavalin, and Barbara Means. Increasing Student Learning Through Multimedia Projects. Alexandria, VA: Assoc. for Supervision and Curriculum Development, 2002.

[10] Sylwester, Robert. A Celebration of Neurons: An Educator's Guide to the Human Brain. Alexandria, VA: Association for Supervision and Curriculum Development, 1995.

[11] Wartella, Ellen and Nancy Jennnings, "Children and Computers: New Technologies- Old Concerns", Children and Computer Technology, Vol. 10. No. 2 / Fall Winter 2000. (http://www.futureofchildren.org)

[12] Woodard, E. and Gridina, M. Media in the home 2000: The fifth annual survey of parents and children. Philadelphia: Annenberg Public Policy Center, University of Pennsylvania, 2000. 